"We should probably see that again; bring it back next week." The "it" was presumably the wound rather than the child, but the woman was upset that she had no time to ask if her child would be all right. In the second case a woman asking questions about her leg ulcer was told, "Look, dear, you go away and make up your mind what you want done, and then we might be able to help you."

When the NHS was founded, people were grateful to have access to free health care and accepted not being treated as equals. But we should be further on in the long march from paternalism. The NHS reforms and the patient's charter are designed to try to make the health service more customer or patient oriented, but structural reforms and exhortations will never be enough on their own. Deeper, cultural changes are needed, and total quality management-with its insistence that only the patient can define quality-is the way forward. This should be familiar to doctors. The first lesson we learn at medical school is to listen to the patient.

RICHARD SMITH

Editor, $B M f$

1 Coulston E. Inquiry urged after sex abuse verdict on doctor. Guardian 1993; February 4:6

2 Clouston E. Victim who protested to health staff was told "not to be silly." Guardian 1993; February $4: 6$.

3 Clouston E. Noises were being made at meetings, parents sounding off . . but the addicts weren't too cut up about it. Guardian 1993; February 4:6.

4 Brindle D. Ombudsman's "worst case" riles MPs. Guardian 1993; February 4:6.

\title{
Antibiotic prophylaxis of neonatal group B streptococcal infections
}

\author{
Rapid testing of women at risk may be worth while
}

The group B streptococcus is the commonest cause of noniatrogenic bacterial sepsis in the first weeks of life in many centres in Britain, continental Europe, Australia, and northern America. ${ }^{2}$ About two thirds of infections occur in the first five days and more than half of these are apparent at birth. These early onset infections are characterised by pneumonia and septicaemia, accompanied in $30 \%$ of cases by meningitis, ${ }^{3}$ and have a mortality of about $20 \%$. The only practicable way of preventing group B streptococcal sepsis is chemoprophylaxis, though immunoprophylaxis may well be an alternative within a decade. ${ }^{5}$ The options available are simple: withhold all chemoprophylaxis, offer it to everyone, or target a risk group. There are difficulties with all three approaches.

A prerequisite for early onset group B streptococcal infection is maternal genital carriage during pregnancy. Reports of genital carriage vary widely, but $20 \%$ to $25 \%$ of women are probably carriers in most countries. Despite this, the incidence of the infection seems to vary widely. In the United States about $1 \%$ of infants born to colonised mothers develop group B streptococcal infection; in northern Europe, including Britain, ${ }^{2}$ the incidence seems to be about 10 times lower. Some European countries do have higher rates-for example, the incidence of group B streptococcal infection in Norway may be as high as 1.8 per 1000 live births. ${ }^{6}$ In England, however, the low infection rate seems to have remained stable over the past decade as the number of cases reported each week to the Communicable Disease Surveillance Centre has not varied greatly.

The heaviness of carriage and the risk of neonatal septicaemia are directly related: infants who are heavily colonised have an increased risk of infection of at least 10-fold..$^{7-9}$ The risk of neonatal group B streptococcal sepsis is also significantly increased in combination with perinatal risk factors. ${ }^{1011}$ In a prospective study in Chicago 61 out of 32384 newborn infants had group B streptococcal septicaemia (1.9 per 1000 live births). When one or more of premature labour, membranes ruptured longer than 18 hours, or intrapartum fever were present the attack rate rose to 7.6 per 1000 , while in the absence of risk factors it fell to 0.6 per 1000 . Fifteen of the 16 cases with a fatal outcome had one or more of these risk factors. ${ }^{10}$

Giving antimicrobial agents to all pregnant women would reduce the incidence of neonatal sepsis due to group $B$ streptococcus but would represent an unjustified overprescription of antibiotics. Indeed, identifying all carriers of group B streptococcus during pregnancy by culture and giving prophylaxis during labour also represents a level of antibiotic administration which is hard to justify. ${ }^{12}$ Some American centres identify carriers and then give prophylaxis during labour to those at increased risk. ${ }^{13}$ This approach is based on the finding that intrapartum ampicillin significantly reduced neonatal group B streptococcal infections when given to carriers who had one or more additional risk factors. ${ }^{13}$ Another reason for adopting this approach is that infants born to mothers who have no perinatal risk factors suffer from less severe infections and respond better to treatment. ${ }^{11} \mathrm{Un}$ fortunately genital carriage of group B streptococcus is unstable, so a swab taken during pregnancy does not necessarily predict carriage at delivery. Combining vaginal and rectal swabs, together with the use of a selective culture medium, improves the predictive value (positive predictive value of $67 \%$ and a negative predictive value of $92 \%{ }^{14}$ ) but only to a level which makes mass screening viable in areas with high rates of infection. In areas of lower incidence the costbenefit ratio of this approach does not justify such large scale screening, and the risk of adverse effects outweighs the benefits. ${ }^{15}$ The fact that group B streptococcal infections are rare in many British hospitals also means that clinicians have little incentive to initiate preventive measures.

An alternative approach to targeting that is particularly suited to low risk areas is offered by rapid tests: first identify the risk during labour and then test for carriage. This avoids mass screening and means that fewer than $5 \%$ of women will receive intrapartum chemoprophylaxis. ${ }^{13}$ Several new kits based on latex particle agglutination or enzyme immunoassay technology can yield results within 20 minutes, and these tests may be performed by the bedside. None are as good as conventional culture, and though their specificity is good the sensitivity is relatively poor (up to $60 \%$ in total but up to $90 \%$ in heavy carriage). ${ }^{16}$ Nevertheless, they seem to be sensitive enough to detect most heavy carriers. ${ }^{18-20}$ In a retrospective analysis a latex agglutination test gave positive results in all mothers who delivered infants with group B streptococcal infections, and no neonatal deaths due to group B streptococcal infection occurred in the group testing negative. ${ }^{21}$ The sensitivity of such kits may also improve.

The choice in Britain is therefore between withholding all chemoprophylaxis and using a rapid screening test. Both decisions involve compromise, but we believe the latter approach should now be taken as it targets a sufficiently small 
group and does not necessitate an expensive mass screening programme. More sensitive conventional cultures should be used when possible. We suggest the following: when an additional risk factor (preterm labour, prolonged rupture of membranes, or fever) is present conventional culture and a rapid test should be performed, and if either gives a positive result antibiotics should be given during labour. In some cases-for example, maternal pyrexia and prolonged rupture of membranes - the rapid test is unnecessary and antibiotics should be given regardless of carriage. Rectal and vaginal cultures should be taken during a multiple pregnancy and if these yield group B streptococcus, prophylaxis should be started during labour. If results are negative a rapid test should be performed during labour. As there are no reliable data on mothers who have already had an infected child, they should receive chemoprophylaxis regardless of culture. It can be argued that such mothers may not develop protective IgG antibodies and may continue to carry the same strain of group B streptococcus. Ampicillin or amoxycillin remain the drugs of choice, ${ }^{9}$ although a first generation cephalosporin may be used in the case of ampicillin allergy. ${ }^{13}$

CARLA VAN OPPEN

Senior Lecturer in Obstetrics and Gynaecology ROBERT FELDMAN

Senior Lecturer in Clinical Microbiology

University Hospital Utrecht,

3508 GA Utrecht,

Netherlands

1 Franciosi RA, Knostman JD, Zimmerman RA. Group B streptococcal neonatal and infan infections. $\mathcal{F}$ Pediatr 1973;82:707-18.
2 Mayon-White RT. The incidence of GBS disease in neonates in different countries. Antibio Chemother 1985;35:17-27.

3 Anthony BF, Okada DM. The emergence of group B streptococci in infections of the newborn infant. Ann Rev Med 1977;28:355-69.

4 Baker CJ. Neonatal sepsis: an overview. In: Morell A, Nydegger U, eds. Clinical use of intravenous immunoglobulins. London: Academic Press, 1986:137-44.

5 Feldman RG, Fleer A. The immune response to the group B streptococcus. Rev Med Microbio 1992;3:52-8.

6 Lindemann R, Henrichsen T, Svenningesen, Hjelle K. Vaginal chlorhexidine disinfection during labour. Lancet 1992;340:792.

7 Gerards LJ, Cats BP, Hoogkamp-Korstanje JAA. Early neonatal group B streptococcal disease: degree of colonisation as an important determinant. F Infect 1985;11:119-24.

8 Pass MA, Gray BM, Khare S, Dillon HC. Prospective studies of group B streptococcal infections in infants. F Pediatr 1979;95:437-43.

9 Dillon HC Jr, Khare S, Gray BM. Group B streptococcal carriage and disease: a 6-year prospective study. I Pediatr 1987;110:31-6.

10 Boyer KM, Gadzala CA, Burd LI, Fisher DE, Paton JB, Gotoff SP. Selective intrapartum chemoprophylaxis of neonatal group B early onset disease. I Epidemiologic rationale. $I$ Infect $D i$ 1983;148:795-801.

11 Schuchat A, Oxtoby M, Cochi S, Sikes RK, Hightower A, Hikaytis B, et al. Population-based risk factors for neonatal group B streptococcal disease: results of a cohort study in metropolitan Atlanta. I Infect Dis 1990;162:672-7.

12 Gibbs RS, Hall RT, Yow MD, McCicken GH, Nelson JD. Consensus: perinatal prophylaxis for group B streptococcal infection. Pediatr Infect Dis $\mathcal{f} 1992 ; 11: 179-83$.

13 Boyer KM, Gotoff SP. Prevention of early-onset neonatal group B streptococcal disease with selective intrapartum chemoprophylaxis. N Engl f Med 1986;314:1665-9.

14 Boyer KM, Gadzala CA, Kelly PD, Burd LI, Gotoff SP. Selective intrapartum chemoprophylaxis of neonatal group B streptococcal early-onset disease. II. Predictive value of prenatal cultures. $\mathcal{J}$ Infect Dis 1983;148:802-9.

15 Easmon CSF, Hastings MJG, Neill J, Bloxham B, Rivers RPA. Is group B streptococcal screening during pregnancy justified? Br f Obstet Gynaecol 1985;92:197-201.

16 Greenspoon JS, Fishman A, Wilcox JG, Greenspoon RL, Lewis W. Comparison of culture for group B streptococcus versus enzyme immunoassay and latex agglutination rapid tests: results in group B streptococcus versus enzyme immunoassay and late

17 Clarke P, Armer T, Duff P, Davidson K. Assessment of a rapid latex agglutination test for group B streptococcal colonisation of the genital tract. Obstet Gynecol 1992;79:358-63.

$18 \mathrm{Lim}$ DV, Morales WJ, Walsh AF, Kazanis D. Reduction of morbidity and mortality rates for neonatal group B streptococcal disease through early diagnosis and chemoprophylaxis. $f$ Clin Microbiol 1986;23:489-92.

19 Morales WJ, Lim DV, Walsh AF. Prevention of neonatal group B streptococcal sepsis by the use of a rapid screening test and selective intrapartum chemoprophylaxis. Am f Obstet Gynecol 1986;155:979-83.

20 Tuppurainen N, Hallman M. Prevention of neonatal group B streptococcal disease: intrapartum detection and chemoprophylaxis of heavily colonized parturients. Obstet Gynecol 1989;73:583-7.

21 Isada NB, Grossman JH. A rapid screening test for the diagnosis of endocervical group B streptococci in pregnancy: microbiologic results and clinical outcome. Obstet Gynecol 1987;70 $139-41$

\section{Richard Doll at 80}

\section{Where there's smoke}

Fifty years ago doctors had recognised that there was a growing epidemic of lung cancer in Western countries but had not identified its origins. The most likely cause was thought to be diesel exhaust fumes. The research published in the $B M F$ in 1950 by Sir Richard Doll and his colleague the statistician the late Sir Austin Bradford Hill solved the problem in a way that was both surprising and conclusive: they showed that cigarette smoking was responsible. ${ }^{1}$ At that time smoking had not been linked with heart disease or even with bronchitis-it was seen as a harmless if foolish habit.

Doll and Bradford Hill had firm opinions on the responsibilities of research workers. It was their job to get results, but they left it to others to act on the research findings. They did not start campaigning against smoking; instead they set out, with popperian zeal, to do more research to challenge their own findings. Their later work looked at the smoking habits and health of doctors and led to the crucial evidence that those who stopped smoking eventually lost their increased risk of lung cancer. ${ }^{2}$

Sir Richard Doll's long and distinguished career as an epidemiologist is to be celebrated on 15 February by a meeting at the Royal Society hosted by the Imperial Cancer Research Fund. He will himself present new data on the cohort of doctors he has been studying for the past 40 years, while his colleagues will review current knowledge on some of the many other topics that he has studied-including the effects on health of oral contraceptives, the parts played by radiation and by asbestos in various cancers, and patterns and trends in mortality. A one day meeting cannot cover all the topics to which Doll has made contributions; his early work in clinical gastroenterology, for example, showed the uselessness of a bland diet for peptic ulcers and also helped establish the importance of basing decisions about treatment on the results of controlled clinical trials.

Readers of the $B M Y$ will remember with pleasure Sir Richard's account of his experiences as a medical officer at Dunkirk, published at the time of the 50th anniversary of the retreat. ${ }^{3} \mathrm{He}$ went on to work in a hospital ship in the Mediterranean-a year which he later described as being one of the most enjoyable of his life. ${ }^{4} \mathrm{He}$ has not lost this capacity for pleasure; by his own account he continues to enjoy his research, and we wish him many more life enhancing years to come.

TONY SMITH

Associate editor $B M \mathcal{F}$

1 Doll R, Bradford Hill AB. Smoking and carcinoma of the lung. BMF 1950;ii:739-48.

2 Doll $\mathrm{R}$ Bradford Hill $\mathrm{AB}$. Mortality in relation to smoking: ten years' observations of Britis doctors. $B M 7$ 7 1964; i $1399-410$

3 Doll R. Experiences of a battalion medical officer in the retreat to Dunkirk. BMF 1990;300:1183-5, 1256-9, 1324-8, 1385-7, 1449-52.

4 Conversation with Sir Richard Doll. Br f Addict 1991;86:365-77. 